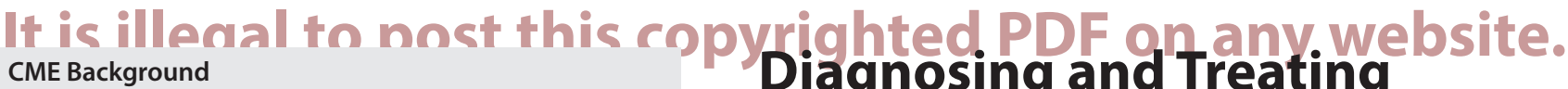 \\ Articles are selected for credit designation based on an assessment of the educational needs of CME participants, \\ Diagnosing and Treating Patients With Mixed Features
} with the purpose of providing readers with a curriculum of CME articles on a variety of topics throughout each volume. Activities are planned using a process that links identified needs with desired results.

To obtain credit, read the article, correctly answer the questions in the Posttest, and complete the Evaluation.

This ACADEMIC HIGHLIGHTS section of The Journal of Clinical Psychiatry presents the highlights of the planning teleconference series "Diagnosing and Treating Patients With Mixed Features," which was held in May and June 2017. This report was prepared and independently developed by the CME Institute of Physicians

Postgraduate Press, Inc., and was supported by an educational grant from Sunovion Pharmaceuticals Inc.

\section{CME Objectives}

After studying this article, you should be able to:

- Use evidence-based screening strategies to recognize signs of mixed features in patients with mood episodes

- Identify patients with risk factors for transition from major depressive disorder to bipolar disorder

- Select treatment for patients with mood episodes and mixed features according to the best available evidence

Accreditation Statement

The CME Institute of Physicians Postgraduate Press, Inc., is accredited by the Accreditation Council for Continuing Medical Education to provide continuing medical education for physicians.

Release, Expiration, and Review Dates This educational activity was published in September 2017 and is eligible for AMA PRA Category 1 Credit $^{\text {TM }}$ through October 31, 2019. The latest review of this material was August 2017.

\section{Financial Disclosure}

All individuals in a position to influence the content of this activity were asked to complete a statement regarding all relevant personal financial relationships between themselves or their spouse/partner and any commercial interest. The CME Institute has resolved any conflicts of interest that were identified. In the past year, Alan J. Gelenberg, MD, Editor in Chief, has been a consultant for Zynx Health, has been a stock shareholder of Healthcare Technology Systems, and has been owner and editor of the Biological Therapies in Psychiatry newsletter. No member of the CME Institute staff reported any relevant personal financial relationships. Faculty financial disclosure appears on the next page.

J Clin Psychiatry 2017;78(8):1091-1102

https://doi.org/10.4088/JCP.su17009ah1c

() Copyright 2017 Physicians Postgraduate Press, Inc.

\author{
Rakesh Jain, MD, MPH; Vladimir Maletic, MD, MS; \\ and Roger S. McIntyre, MD, FRCPC
}

$\mathbf{P}$ atients who present with major depressive disorder (MDD) and subthreshold hypomania are often misdiagnosed. Using older criteria, clinicians have diagnosed such patients as having MDD alone or bipolar disorder. Newer diagnostic criteria allow for a diagnosis of MDD with a mixed features specifier. Patients with MDD and subthreshold hypomania are at increased risk of developing bipolar disorder and must be followed carefully. Treating patients with mixed features is challenging, but treatment guidelines have emerged.

This ACADEMIC Highlights offers summaries of presentations by experts on mixed features. The Chair, Rakesh Jain, MD, MPH, discussed screening patients for mixed features and applying the DSM-5 specifier. Vladimir Maletic, MD, MS, addressed the identification of patients with unipolar depression who may be at increased risk for progression to bipolar disorder. Roger S. McIntyre, MD, FRCPC, reviewed how to tailor patients' treatment regimens for mood episodes with mixed features.

\section{ORIGINS AND EVOLUTION OF THE CONCEPT OF MIXED STATES}

Dr Jain began by noting that the concept of mixed states has a long history. Descriptions of mixed states date back to classical times and can be found in the writings of ancient Greek physicians such as Hippocrates and Aretaeus of Cappadocia, both of whom espoused the existence of melancholia and mania. ${ }^{1}$ The ancient Greek scholars had a broader concept of these terms than is currently accepted, but their ideas still roughly correspond to the modern concepts of depressive and manic states. These classical physicians acknowledged that individuals could experience and switch between discrete mood states, but they could also experience a mixture of both melancholia and mania. ${ }^{1}$

Eighteenth century scholars rekindled interest in the idea of mixed mood states, but the current concept of mixed states dates to the end of the 19th century. ${ }^{1}$ The German psychiatrist Emil Kraepelin introduced the term "mixed state" in 1896. ${ }^{1,2}$ Remarking that Kraepelin is often said to have "launched a thousand ships" in modern psychiatry, Dr Jain commented that Kraepelin's work is still relevant today.

One of Kraepelin's students and colleagues, Wilhelm Weygandt, published the first book on mixed states in 1899 and helped develop the concept. ${ }^{2}$ Kraepelin further refined the concept of mixed states, eventually defining 6 distinct types. ${ }^{1}$ According to Kraepelin, the 3 main symptoms of mania were flight of ideas, euphoria, and hyperactivity, and the 


\section{It is illenal to nost this cony} Credit Designation

The CME Institute of Physicians Postgraduate Press, Inc. designates this journal-based CME activity for a maximum of 1 AMA PRA Category 1 Credit $^{\text {TM }}$. Physicians should claim only the credit commensurate with the extent of their participation in the activity.

Note: The American Academy of Physician Assistants (AAPA) accepts certificates of participation for educational activities certified for AMA PRA Category 1 Credit $^{\text {TM }}$ from organizations accredited by ACCME or a recognized state medical society. Physician assistants may receive a maximum of 1 hour of Category I credit for completing this program.

The teleconference was chaired by Rakesh Jain, MD, MPH from the Department of Psychiatry, Texas Tech University Health Sciences Center Medical School, Midland. The faculty members were Vladimir Maletic, MD, MS, from the Department of Neuropsychiatry and Behavioral Sciences, University of South Carolina School of Medicine, Greer; and Roger S. McIntyre, MD, FRCPC, from the Mood Disorders Psychopharmacology Unit, University of Toronto, Ontario, Canada.

\section{Financial Disclosure}

Dr Jain is a consultant for Addrenex, Allergan, Eli Lilly, Lundbeck, Merck, Otsuka, Pamlab, Pfizer, Shionogi, Shire, Sunovion, and Takeda; has received grant/research support from AstraZeneca, Allergan, Eli Lilly, Lundbeck, Otsuka, Pfizer, Shire, and Takeda; and is a member of the speakers/advisory boards for Addrenex, Alkermes, Allergan, Forum, Eli Lilly, Lundbeck, Merck, Otsuka, Pamlab, Pfizer, Rhodes, Shionogi, Shire, Sunovion, and Takeda. Dr Maletic is a consultant for Lundbeck, Merck, Otsuka, Sunovion, Supernus, Takeda, and Teva and has received honoraria from and is a member of the speakers/advisory boards for Allergan, Lundbeck, Merck, Otsuka, Sunovion, and Takeda. Dr McIntyre has received speakers/consultation fees from Shire, Purdue, Otsuka, Janssen-Ortho, Lundbeck, Pfizer, Neurocrine, Neuralstem, Sunovion, Takeda, and Allergan and has received research support from Lundbeck, Shire, Purdue, Allergan, and Stanley Medical Research Institute.

The opinions expressed herein are those of the faculty and do not necessarily reflect the opinions of the CME provider and publisher or the commercial supporter.

fundamental symptoms of depression were inhibition of thought, depressive mood, and weakness of volition. ${ }^{1}$ Thus, mixed mood states could arise if one of the symptoms of mania was replaced with a symptom of depression, or vice versa. Kraepelin also acknowledged that mixed states could exist either as a transitional form, when a patient is switching from one mood state to another, or as an independent and distinct mood state. $^{1}$

Kraepelin's conceptualization of mood disorders was challenged in the 20th century by the work of Kurt Schneider, who denied the existence of mixed states and claimed that any condition that appeared to combine manic and depressive symptoms should not be considered a mood disorder. ${ }^{2}$ With the publication of the third edition of the Diagnostic and Statistical Manual of Mental Disorders (DSM-III) in 1980, mood disorders were split into bipolar disorder and MDD, thus rejecting Kraepelin's dimensional construct and causing mixed states to be largely forgotten for many years. ${ }^{3}$ Dr Jain noted that although patients could be diagnosed as experiencing a mixed episode, they had to meet full diagnostic criteria for both a manic episode and a depressive episode for at least 1 week. $^{4}$

The dichotomy established by DSM-III ${ }^{4}$ and maintained through $D S M-I V-T R^{5}$ was somewhat problematic because the reality of mood disorder presentations was far more complex ${ }^{6}$ and the experience of mixed episodes is quite common. ${ }^{7}$ To remedy this problem, the $D S M-5^{8}$ introduced the "mixed features" specifier, thus shifting from a categorical conceptualization of mood disorders to more of a dimensional spectrum. People diagnosed with manic, hypomanic, or depressive episodes can meet criteria for the mixed features specifier. ${ }^{8}$ This change acknowledged the prevalence and importance of the subsyndromal mixed states often experienced by individuals with mood disorders (Figure 1). ${ }^{9}$ In looking at this figure, Dr Jain observed that the older DSM construct seemed to consist of islands. The islands of manic, mixed, and depressed episodes each had a large gulf between them. But the newer concept is that mood disorders are much more on a spectrum, like a continent on which individuals could start at one end and go to the other. The landscapes change, but the landscape changes can be gradual. The newer depiction of mixed features shows that they can intrude in every phase of the condition.

\section{EPIDEMIOLOGY AND IMPACT OF MIXED FEATURES}

Dr Jain pointed out a growing awareness of the prevalence of and additive burden created by mixed presentations. For instance, Bauer and colleagues ${ }^{10}$ assessed 441 individuals being treated for bipolar disorder and found that $94.1 \%$ of the patients with mania or hypomania were also experiencing clinically significant depressive symptoms, and $70.1 \%$ of the patients who were in a depressive episode were experiencing clinically significant manic symptoms. Similarly, in a group of 76 patients with MDD, Benazzi ${ }^{11}$ found an average (mean) of 2.8 hypomanic symptoms per patient.

The prevalence of patients meeting the mixed features specifier has varied in study populations. A study of 2,811 patients experiencing a major depressive episode ${ }^{12}$ found a $7.5 \%$ rate of patients meeting criteria for the mixed features specifier. In a post hoc analysis of data from the International Mood Disorders Collaborative Project, ${ }^{13}$ the DSM-5 mixed features specifier criteria were met by $32 \%$ of 573 individuals 
It is illegal to post this convriohted PDE on any website.

Figure 1. Conceptualization of Bipolar Mixed States in DSM-IV-TR ${ }^{5}$ Versus $D S M-5^{8}$ Editions ${ }^{a}$

\begin{tabular}{|c|c|c|c|c|c|}
\hline $\begin{array}{l}\text { Core } \\
\text { symptoms }\end{array}$ & $\begin{array}{c}\text { Elevated } \\
\text { mood }\end{array}$ & \multicolumn{3}{|c|}{$\begin{array}{l}\text { Elevated mood }+ \\
\text { depressed mood or loss of interest }\end{array}$} & $\begin{array}{l}\text { Depressed mood } \\
\text { or loss of interest }\end{array}$ \\
\hline$\overline{M a n i c}$ & $\geq 3$ & \multirow{2}{*}{\multicolumn{3}{|c|}{$\begin{array}{l}\geq 3 \\
\geq 5\end{array}$}} & $\begin{array}{c}<3 \\
\end{array}$ \\
\hline Depressive & $<5$ & & & & $\geq 5$ \\
\hline$D S M-I V-T R$ & Manic & \multicolumn{3}{|c|}{ Mixed } & Depressive \\
\hline DSM-5 & Manic & \multicolumn{2}{|c|}{ Manic with mixed features } & $\begin{array}{l}\text { Depressive with } \\
\text { mixed features }\end{array}$ & Depressive \\
\hline $\begin{array}{l}\text { Core } \\
\text { symptoms } \\
\end{array}$ & $\begin{array}{c}\text { Elevated mood + } \\
\text { energy } \\
\end{array}$ & \multicolumn{2}{|l|}{$\begin{array}{c}\text { Elevated mood }+ \\
\text { energy }\end{array}$} & $\begin{array}{l}\text { Depressed mood } \\
\text { or loss of interest }\end{array}$ & $\begin{array}{l}\text { Depressed mood } \\
\text { or loss of interest }\end{array}$ \\
\hline $\begin{array}{l}\text { Manic } \\
\text { Depressive }\end{array}$ & $\begin{array}{l}\geq 3 \\
<5\end{array}$ & \multicolumn{2}{|l|}{$\begin{array}{l}\geq 3 \\
\geq 3\end{array}$} & $\begin{array}{l}\geq 3 \\
\geq 5\end{array}$ & $\begin{array}{l}<3 \\
\geq 5\end{array}$ \\
\hline $\begin{array}{l}\text { a Reprinted } \mathrm{W} \\
\text { Abbreviation } \\
\text { DSM-5=Dia }\end{array}$ & $\begin{array}{l}\text { ith permission } f \\
\text { s: DSM-IV-TR }=D \\
\text { gnostic and Stat }\end{array}$ & $\begin{array}{l}\text { lu et al. }{ }^{9} \\
\text { stic and Statis } \\
\text { Manual of Me }\end{array}$ & $\begin{array}{l}\text { al of M } \\
\text { ers, Fif }\end{array}$ & Hers, Fourth Editic & xt Revision; \\
\hline
\end{tabular}

Figure 2. Prevalence of DSM-5 Mixed Features Specifier in Adults With Major Depressive Disorder or Bipolar Disorder ${ }^{a}$

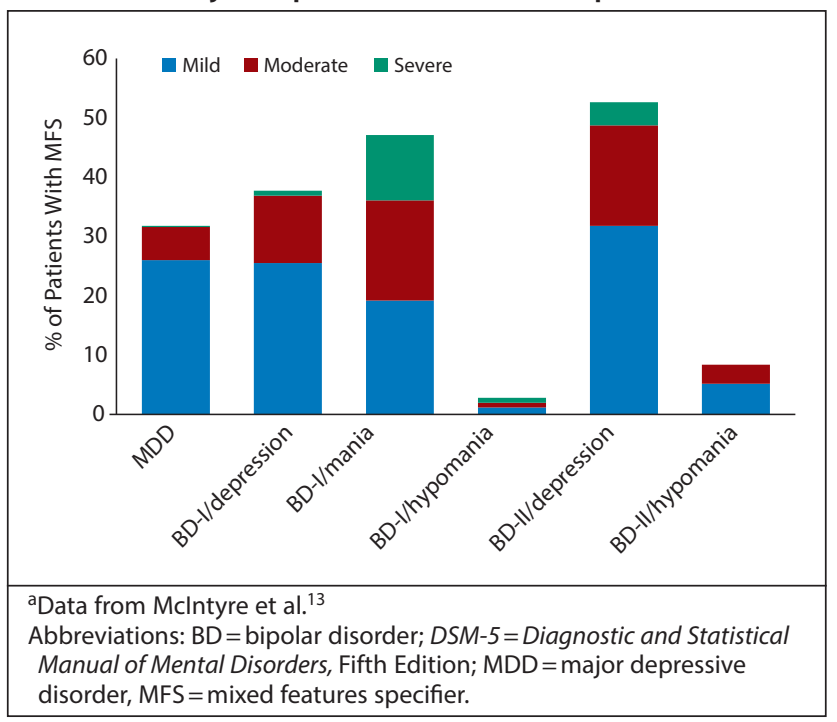

diagnosed with an index major depressive episode

(Figure 2).

Compared with individuals who experience episodes of pure mania or depression, those who experience mixed states have been found to experience a worse clinical course, higher rates of comorbidities, and a poorer response to treatment. ${ }^{2}$ For example, a study of nearly 600 patients with recurrent MDD (and no known family history of bipolar disorder) ${ }^{14}$ found that nearly 1 in 10 had a history of subthreshold manic symptoms. These patients were significantly more likely to have a history of poor response to antidepressants $(P<.02)$, a greater likelihood of experiencing psychosis $(P<.04)$, more depressive episodes $(P=.006)$, and more hospitalizations $(P<.03) .{ }^{14}$ The new mixed features specifier in DSM-5 can help clinicians identify patients such as these, whose symptoms may have been missed with the previous diagnostic criteria.

\section{SCREENING PATIENTS FOR MIXED FEATURES}

Because the spectrum of mood disorders is much larger, wider, and more damaging than once thought, Dr Jain recommended that every patient diagnosed with a mood episode be evaluated for symptoms of the other pole (ie, depressive versus manic/hypomanic). ${ }^{15}$ Some patients may present with red flags, such as MDD with high rates of comorbid anxiety, ${ }^{15}$ high levels of irritability and mood lability, distractibility, and psychomotor agitation (as indicated by such behaviors as pacing, hand wringing, or inability to sit still). ${ }^{12,16}$ Other common symptoms include pressured speech, impulsivity, hyperactivity, and racing thoughts. ${ }^{12,16}$ However, even in the absence of red flags, patients should be assessed for mixed features. Assessment should include questions about mood shifts immediately before or after previous episodes of depression and about unusual periods of enhanced function. Any temporal relationships between mood changes and antidepressant treatment should also be investigated.

Assessment tools can greatly assist in the detection of mixed symptom presentations. Guidelines ${ }^{15}$ suggest using the Quick Inventory of Depressive Symptomatology ${ }^{17}$ self-report scale or the Clinically Useful Depression Outcome Scale ${ }^{18}$ to screen patients for depressive symptoms.

Tools for screening patients for manic/hypomanic symptoms include the Clinically Useful Depression Outcome Scale-Mixed ${ }^{19}$ and the Bipolar Depression Rating Scale. ${ }^{15,20}$ The 2 screening tools that Dr Jain most highly recommended are the Mood Disorder 
Diagnostic Scale (BSDS).

The MDQ is a brief self-report instrument that screens for a lifetime history of manic or hypomanic symptoms. ${ }^{21}$ The MDQ includes 13 yes/no questions to determine the presence of manic/hypomanic symptoms, as well as questions to determine the timing of their occurrence and any impairment associated with the symptoms. ${ }^{22}$ The MDQ has been found to have good sensitivity and specificity for detecting bipolar disorder, but modified scores may be needed for detecting symptoms of mania/ hypomania in patients with $\mathrm{MDD}^{23}$

The BSDS is another useful screening tool that was developed to detect milder presentations of bipolar symptoms such as those characteristic of bipolar II disorder or not otherwise specified. ${ }^{24}$ The BSDS is a self-report tool consisting of 2 parts. In the first part, the respondent is asked to read a paragraph consisting of 19 sentences that describe many of the symptoms of bipolar disorder, including symptoms of both mania and depression. Respondents checkmark each sentence that they believe applies to them. The second part of the assessment is a single multiple choice question asking respondents the extent to which they feel the paragraph describes them overall, with the answers ranging from perfectly to not at all. The BSDS has been found to be more effective than the MDQ for detecting milder signs of mania/hypomania, while the MDQ is effective in detecting bipolar I disorder. ${ }^{24}$ Therefore, employing both scales may be a useful strategy to screen patients for the criteria in the mixed features specifier. $^{24}$

If possible, collateral histories should be obtained from significant others, family members, or friends. Dr Jain commented that these additional sources of information can considerably improve the detection of mixed features. As part of the psychiatric evaluation, information from collateral sources can provide useful information about past or current symptoms and changes in behavior or functioning. ${ }^{25}$

Finally, Dr Jain stated, the DSM- $5^{8}$ mixed features specifier criteria should be used to confirm the diagnosis.

\section{APPLYING THE DSM-5 SPECIFIER}

The DSM-5 provides specific criteria for identifying mixed features in patients with a primary diagnosis of bipolar disorder or MDD. ${ }^{8}$ In order to reduce the potential for overdiagnosis due to overlapping symptoms, some symptoms that can be included in the diagnosis of a manic/ hypomanic or a depressive episode are excluded from the criteria for mixed features. These symptoms include irritability, distractibility, and psychomotor agitation, which are common to episodes of both depression and mania. ${ }^{2}$

\section{Manic or Hypomanic Episode With Mixed Features}

Patients who meet the criteria for a manic or hypomanic episode should receive the mixed features specifier if they

have at least 3 of the following 6 depressive symptoms: (1) prominent dysphoria or depressed mood, (2) psychomotor retardation, (3) feelings of worthlessness or excessive guilt, (4) diminished interest or pleasure in almost all activities, (5) fatigue or loss of energy, or (6) recurrent thoughts of death. ${ }^{8}$ The symptoms should occur most of the time during the current or most recent manic/hypomanic episode. These symptoms should represent a change from usual behavior and should be observable by others.

\section{Depressive Episode With Mixed Features}

The mixed features diagnostic criteria are the same for depressive episodes, regardless of whether the patient's diagnosis is bipolar disorder or MDD. ${ }^{8}$ To receive the mixed features specifier, the patient must have 3 or more of the following symptoms of mania/ hypomania: (1) elevated or expansive mood, (2) inflated self-esteem or grandiosity, (3) increased talkativeness or pressured speech, (4) flight of ideas or racing thoughts, (5) increase in energy or goal-directed activity, (6) increased or excessive involvement in risky behavior, or (7) decreased need for sleep. ${ }^{8}$ The symptoms should occur most of the time during the depressive episode, represent a change from usual behavior, and should be observable by others.

\section{Case Practice Question}

Discussion of the best response can be found at the end of the activity.

Case 1. Juan is a 27-year-old father of 2 with a history of bipolar II disorder. He presents because of his wife's concerns about his anger and irritability, and he also reports insomnia and racing thoughts. You suspect he is experiencing a hypomanic episode but want to screen for mixed features. The presence of which of the following symptoms could indicate that Juan meets the mixed features specifier criteria?

a. Irritability

b. Distractibility

c. Sad mood

d. Decreased need for sleep 


\section{CONCLUSION}

The DSM-5 mixed features specifier provides a much broader and less restrictive diagnostic framework than in previous editions of the DSM that is intended to more accurately represent the spectrum of mood disorder presentations. ${ }^{26}$ The use of the specifier offers the opportunity to recognize subsyndromal symptoms that can have an important impact on treatment. ${ }^{2}$ Dr Jain concluded his presentation by remarking that many clinicians, ${ }^{2}$ including himself, have missed a patient's mixed features, and improving one's awareness and use of the specifier is an important step in providing patient care that leads to better outcomes.

\section{IDENTIFYING PATIENTS WITH UNIPOLAR DEPRESSION AT RISK FOR PROGRESSION TO BIPOLAR DISORDER}

To begin his presentation, Dr Maletic noted that differentiation of mood disorders based on a descriptive classification continues to be a daunting clinical challenge. Citing a study ${ }^{27}$ of 108 patients with depression and/ or anxiety, he pointed out that one-third of those with depression were found to have a bipolar spectrum disorder. Furthermore, Dr Maletic noted that interrater reliability is low for the diagnosis of MDD. ${ }^{28}$ Part of the difficulty in accurately diagnosing unipolar depression is that this diagnosis encompasses a vast range of combinations of symptoms, which can lead to unreliable or inconsistent diagnostic assessments. ${ }^{28,29}$ Additionally, patients often underreport or fail to report hypomanic/manic symptoms, which hinders clinicians' ability to diagnose mixed features or bipolar disorder. ${ }^{30}$ In fact, one survey found that patients were more likely to not report than to report elated mood, increased activity, or reckless behavior to their providers. ${ }^{30}$

The reconceptualization of mood disorder diagnoses in $D S M-5$ was intended to reduce the number of diagnostic errors, but the potential for misdiagnosis still exists. ${ }^{9,26,29}$ In addition to using the diagnostic criteria to diagnose mixed features in major depressive episodes, clinicians can improve their ability to recognize patients with mixed features who are at risk for progression to bipolar disorder. Dr Maletic cited a recent meta-analysis ${ }^{31}$ showing that almost one-fourth of adolescents and adults with MDD who were followed for a mean duration of 12 to 18 years developed bipolar disorder, usually within the first 5 years. Accurate detection of the full range of a patient's symptoms, particularly mania or hypomania, is imperative in patients with depression because the experience of mixed states ${ }^{32}$ - even subthreshold hypomanic symptoms - can indicate a risk of conversion to bipolar disorder. $^{15}$

Epidemiologic and clinical features can both complicate and aid identification of individuals with depression who might have or might be at risk for bipolar disorder. Depression is the dominant mood phase for patients with either bipolar I or bipolar II disorder. Dr Maletic highlighted classic studies by Judd and colleagues on the weekly symptomatic status of patients with bipolar $\mathrm{I}^{33}$ and bipolar $\mathrm{II}^{34}$ disorders. They found that patients with bipolar I disorder spent $31.9 \%$ of the time experiencing depressive symptoms, ${ }^{33}$ while patients with bipolar II disorder spent $50.3 \%$ of the time experiencing depressive symptoms. ${ }^{34}$ Therefore, Dr Maletic noted, these patients will most likely present to their clinicians during a major depressive episode. If these patients receive only a crosssectional assessment, they are likely to receive a diagnosis of MDD. ${ }^{29}$ Careful evaluation will be necessary to detect past or current symptoms of mania or hypomania that might indicate a diagnosis of either mixed features or bipolar disorder.

\section{Risk Factors}

Several clinical indicators are known to be more strongly associated with bipolar disorder than with MDD. Dr Maletic described risk factors for bipolar disorder in patients experiencing episodes of depression (Table 1). ${ }^{15,31,35-37}$

Early age at onset. Individuals with bipolar disorder tend to experience an earlier age at onset compared with individuals with MDD. ${ }^{36}$ Dr Maletic described a study by Oedegaard and colleagues ${ }^{38}$ (Figure 3), in which twice as many patients with bipolar disorder (13\%) had onset of depression before age 13 years compared to those with MDD (6\%). While 19\% of those with MDD had an onset during ages 13 to 19 years, $48 \%$ of those with bipolar disorder did. During ages 20 to 29 years, the likelihood of a bipolar disorder or MDD onset was equivalent. Past the age of 30 years, onset of depression was 4 times more likely to be associated with MDD than with bipolar disorder.

Table 1. Risk Factors for Progression From

Unipolar Depression to Bipolar Disorder ${ }^{a}$

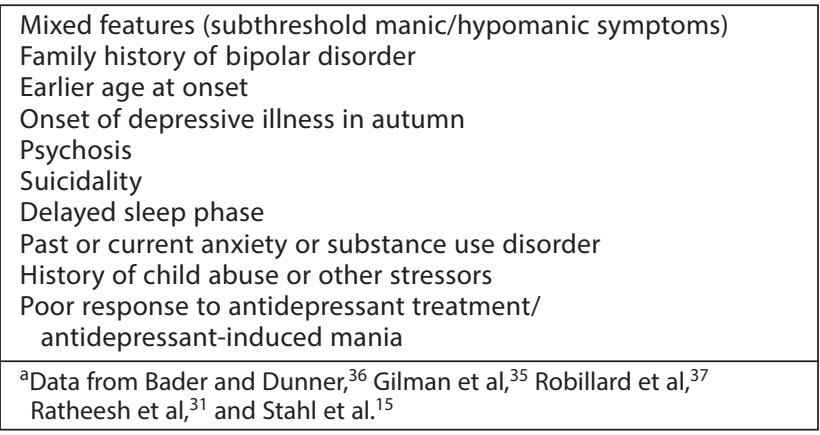


It is illegal to nost this con Figure 3. Age at Onset of Bipolar Disorder Versus Major Depressive Disorder ${ }^{\mathrm{a}}$

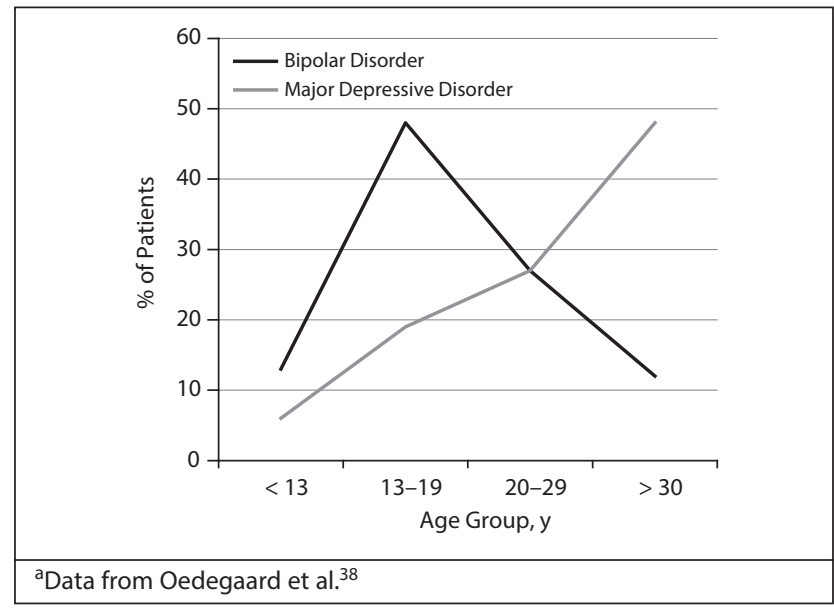

Female sex. Sex differences in bipolar disorder may delay correct diagnosis. Women are less likely than men to experience mania at the onset of bipolar I disorder. ${ }^{39}$ Dr Maletic commented, therefore, that weighing the risk of bipolar disorder is particularly important in female patients presenting with an early-onset depressive episode.

Season of first episode. Interestingly, even the time of year of a patient's first depressive episode can be informative. The peak season for initial episodes of unipolar depression (without mixed features) has been found to be spring, whereas the peak season for the onset of bipolar depression or depression with mixed features is fall. $^{40}$

Symptom differences. Symptom presentation can provide indications as to the likelihood of a patient with mixed features converting to bipolar disorder. Sleep disturbance is a common feature of both MDD and bipolar disorder, and circadian rhythm disruption is believed to play a causal role in the pathophysiology of mood disorders. ${ }^{41}$ Sleep difficulties may manifest differently depending on the disorder. Robillard and colleagues ${ }^{37}$ found that patients experiencing an episode of bipolar depression were significantly more likely to exhibit a delayed sleep phase than individuals experiencing an episode of unipolar depression $(P=.014)$.

Other relevant symptoms are psychosis and suicidal ideation, which become more common in patients with unipolar depression as their number of manic/hypomanic symptoms increase. ${ }^{42}$ Dr Maletic pointed out a study by Goldberg et al, ${ }^{43}$ who found that patients who presented with psychotic symptoms during an initial major depressive episode were significantly more likely than those without initial psychosis to subsequently develop mania $(P=.003)$. This research finding was supported by Tohen and colleagues ${ }^{44}$ who found that within a 2 -year follow-up of patients with first-episode psychotic depression, 33\% met criteria for a bipolar disorder diagnosis.

Dr Maletic also described the relationship between suicidality, MDD with mixed features, and bipolar disorder.
Figure 4. Bipolar Disorder Among First-Degree Relatives in Patients With Major Depressive Disorder Versus Bipolar Disorders ${ }^{\mathrm{a}}$

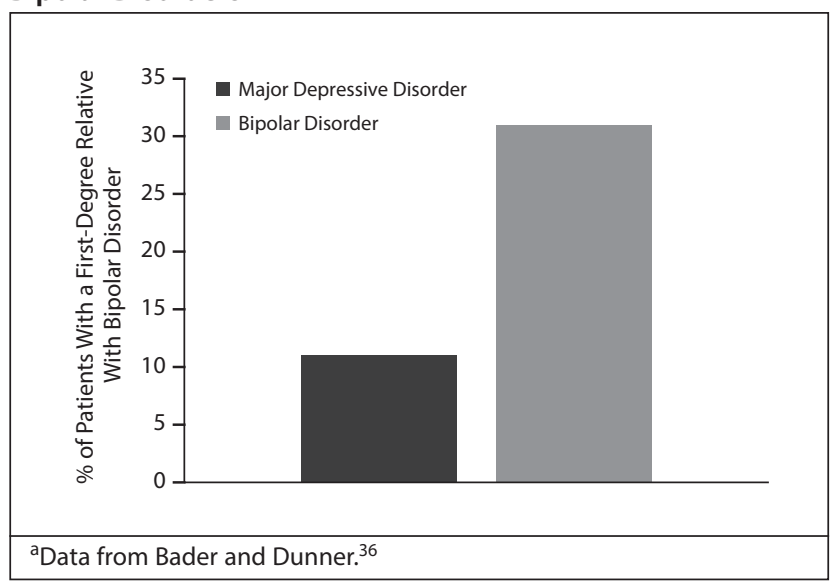

In a retrospective claim review, $M c I n t y r e ~ e t ~ \mathrm{al}^{45}$ found that, compared with individuals with MDD only, those with MDD and mixed features were 5 times more likely to experience suicidal ideation $(\mathrm{OR}=4.9)$ and 3 times more likely to engage in suicidal behavior $(\mathrm{OR}=2.8)$, both of which were statistically significant increases $(P<.001)$. Furthermore, a study by Akiskal and Benazzi ${ }^{46}$ found that the experience of racing/crowded thoughts and psychomotor agitation/activation both independently increased the likelihood of suicidal ideation in patients with MDD. A retrospective chart review ${ }^{36}$ showed a higher rate of suicide attempts among patients with bipolar I disorder (42\%) than among those with recurrent MDD (16\%).

Psychiatric history. The patient's current and past psychiatric diagnoses can reveal possible risk factors for conversion to bipolar disorder. Gilman and colleagues ${ }^{35}$ used data from the National Epidemiologic Survey on Alcohol and Related Conditions to predict conversion to bipolar disorder over the course of 3 years in 6,214 individuals initially diagnosed with MDD. They found that patients with a history of social phobia or generalized anxiety disorder were significantly more likely to transition to a diagnosis of bipolar disorder $(P<.05$ for both disorders).

Current comorbidities are also important considerations. A 10 -year study ${ }^{47}$ found that patients with MDD and mixed features (subthreshold manic symptoms) were more likely than those without subthreshold mania to convert to bipolar disorder; this group of patients also had higher rates of alcohol use disorder and panic disorder.

Stressors. Lifetime history of stressors should be assessed. Stressful life events, including past experience of child abuse as well as recent problems with social support, such as loss or injury of a close friend or family member or a separation or divorce, have been found to increase the likelihood of a transition to a bipolar disorder diagnosis. ${ }^{35}$

Family history. A retrospective chart review of patients with bipolar spectrum disorders or MDD was conducted by Bader and Dunner (Figure 4). ${ }^{36}$ They found that 
significantly more likely than those with MDD to have a first-degree relative with bipolar disorder (27\%-32\% vs $11 \%$, respectively; $P<.05)$. This insight into the genetic underpinnings and heritability of bipolar disorders underscores the importance of obtaining a thorough family history in all patients with mood symptoms. A family history of bipolar disorder could be considered a risk factor for conversion to bipolar disorder in a patient with depressive symptoms.

Antidepressant use and response. Antidepressant treatment has been found to be associated with an increased risk of transition to bipolar disorder in disposed individuals with MDD. In a review of 51 reports, Baldessarini and colleagues ${ }^{48}$ found a rate of switching to bipolar disorder of $8.18 \%$ (or $3.42 \%$ per year) among patients with MDD who were receiving antidepressant treatment. Dr Maletic pointed out that, in 10 controlled trials, the incidence of conversion was 2.6 times greater in those taking antidepressants than in those not taking antidepressants. ${ }^{48}$

Past response to antidepressant treatment may predict conversion to bipolar disorder. Li and colleagues ${ }^{49}$ found a strong association between poor antidepressant response and a conversion from a diagnosis of MDD to bipolar disorder. This study evaluated data from 2 large cohorts of individuals diagnosed with MDD over 8 years. The patients who experienced poor response to antidepressant treatment were significantly more likely to convert to bipolar disorder than those who showed a favorable response to treatment $(P<.0001) .{ }^{49}$ Thus, poor response to antidepressant treatment should be considered as one of the many possible clinical factors that can signal a patient's risk of converting to bipolar disorder.

\section{Case Practice Question}

Discussion of the best response can be found at the end of the activity.

Case 2. Anthony has had recurrent depression since the autumn that he began high school. He is now 28 years old and experiencing another depressive episode. He has failed 3 adequate trials of antidepressants. Anthony is currently experiencing intense anxiety and sleep disturbance. Besides young age and seasonality at onset and poor antidepressant response, which other clinical feature represents a risk that Anthony may transition from MDD to bipolar disorder?
a. Middle insomnia
b. Cognitive difficulties
c. Family history of anxiety disorders
d. History of frequent suicidal thoughts

\section{CONCLUSION}

In summary, Dr Maletic offered some clinical pearls. Mood disorders cannot be easily classified on the basis of a cross-sectional evaluation. Rather, clinicians who evaluate patients reporting a depressive episode need to obtain a detailed history, including family psychiatric history, and conduct a careful longitudinal follow-up with a specific focus on the emergence of hypomanic or manic symptoms throughout the course of illness. It is helpful to remember that mood disorders are dynamic, evolving conditions, and a diagnosis that seemed accurate at one point may no longer be accurate a few years later.

\section{TAILORING PATIENTS'TREATMENT REGIMENS FOR MOOD EPISODES WITH MIXED FEATURES}

A guiding principle of medicine, began Dr McIntyre, is primum non nocere, or "first, do no harm." Of course, all treatments carry risks of adverse effects, but evidence ${ }^{50}$ suggests that the use of antidepressants for a depressive episode may destabilize not only patients with bipolar disorder but also those with unipolar depression and mixed features. How should clinicians safely and effectively treat patients who have depressive episodes with mixed features? In his presentation, Dr McIntyre addressed this question and described recent guidelines.

Dr McIntyre began by pointing out a real-world assessment of the challenges faced by these patients in treatment settings. He and his colleagues ${ }^{45}$ retrospectively reviewed the medical claims of over 130,000 individuals with newly diagnosed MDD during the year after the diagnosis. The claims were from a commercial claims database from January 1, 2009, to October 31, 2014. This study found that, compared to patients with MDD only, those with MDD and mixed features appeared to have a more difficult-to-treat illness as evidenced by a greater need for polypharmacy with multiple drug classes, more psychotherapy, and significantly higher rates of mental health-related emergency department visits, outpatient visits, and inpatient stays $(P<.001$ for all). Additional evidence ${ }^{2,9,15,51}$ indicates that patients who experience depression with mixed features often exhibit a more complex illness presentation, higher rates of recurrence and chronicity, and poorer response to antidepressant treatment than those without mixed features, meaning that these patients will require special treatment consideration.

Unfortunately, compared with other manifestations of mood disorders, depression with mixed features does not have a strong evidence base to guide treatment selection due to the relatively limited number of clinical trials that have been conducted in this area. In 2015, the Florida Medicaid program drew on the best available evidence and released a practice guideline ${ }^{52}$ that included 


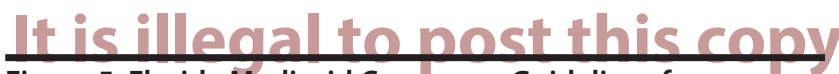

Figure 5. Florida Medicaid Consensus Guidelines for Pharmacologic Treatment of Major Depressive Disorder With Mixed Features ${ }^{\mathrm{a}}$

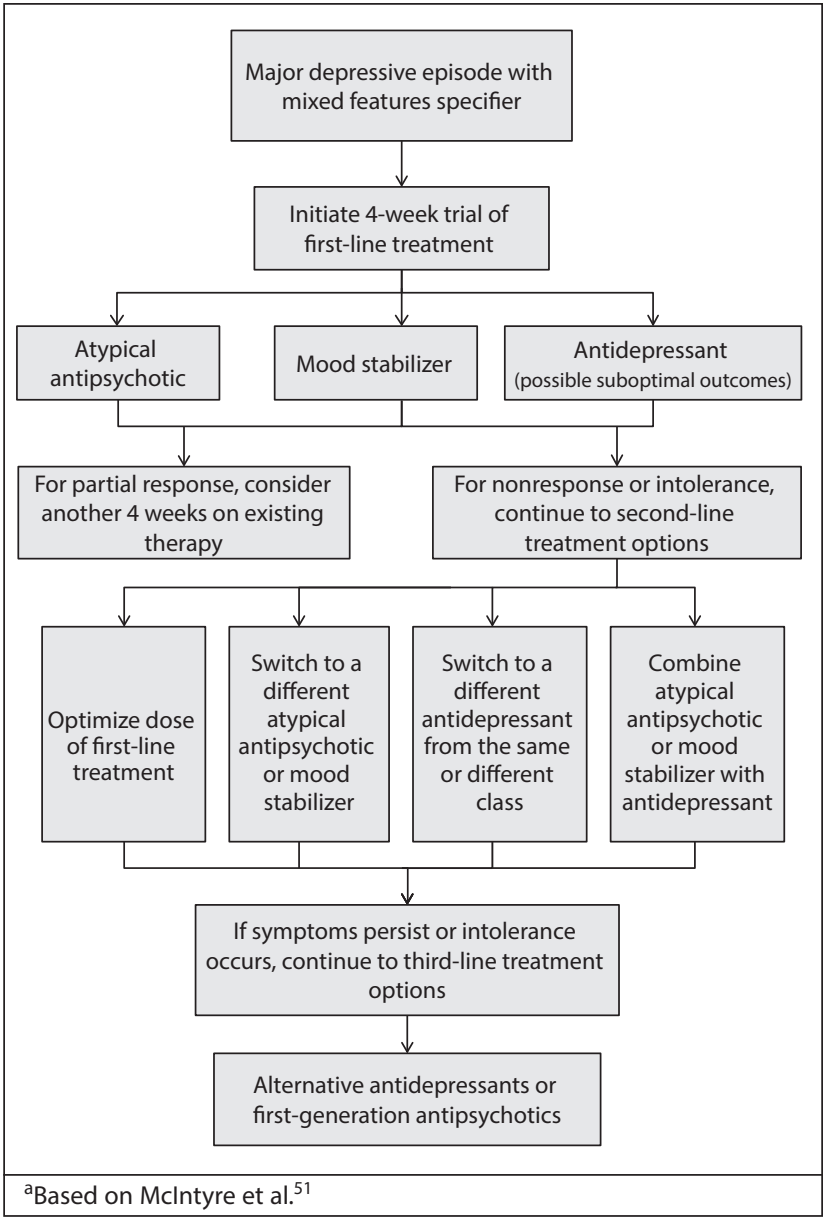

the first available treatment algorithm for MDD with mixed features as defined by the DSM-5. The treatment recommendations from this guideline were recently updated (Figure 5). ${ }^{51}$ An additional set of guidelines for the recognition and management of mixed depression was developed by a panel of mood disorder experts based on the DSM-5 diagnostic criteria, the best available research, and the panel's clinical expertise (Figure 6). ${ }^{15}$

\section{Pharmacologic Treatment}

Dr McIntyre emphasized that, before beginning pharmacologic treatment in any patient with a major depressive episode who is exhibiting symptoms sufficient to meet the criteria for the mixed features specifier, the patient must be carefully evaluated for a current or past history of mania or hypomania in order to rule out a diagnosis of bipolar disorder. Patients should also be assessed for other psychiatric or medical comorbidities that could be contributing to mixed features. A variety of conditions from substance use to obesity have been found to be associated with symptoms similar to those of mixed features, including irritability, impulsivity, and mood dysregulation. ${ }^{15,51}$
Figure 6. Expert Consensus Recommendations for Pharmacologic Treatment of Major Depressive Disorder With Mixed Features ${ }^{\mathrm{a}}$

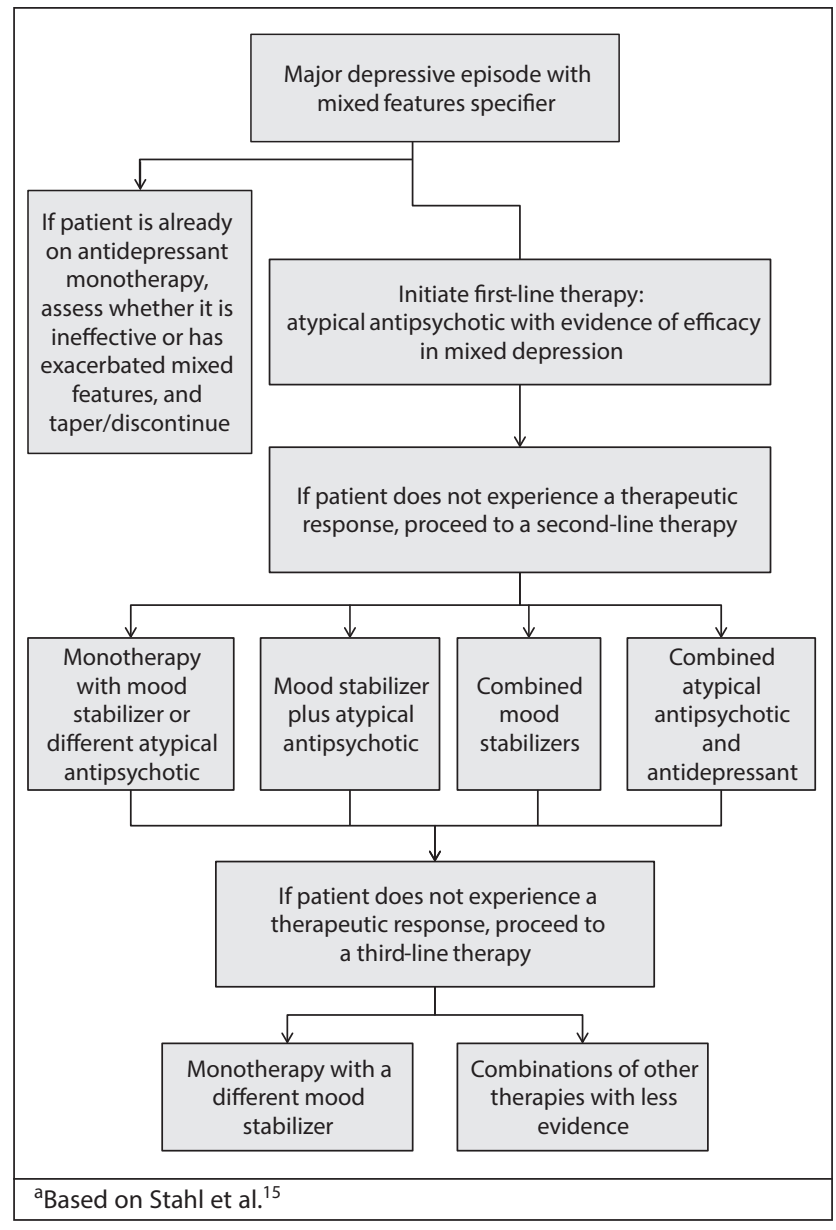

For patients with a diagnosis of depression with mixed features according to the DSM- 5 criteria, the Florida guidelines are similar to the guidelines for treating MDD without mixed features. ${ }^{51}$ Although the guidelines acknowledge that mood destabilization is a risk with antidepressant treatment in patients experiencing manic or hypomanic symptoms, the panel concluded that not enough evidence was available in patients with MDD and DSM-5-defined mixed features to forbid the use of conventional antidepressants. Thus, antidepressant monotherapy is endorsed as one firstline treatment option for major depression with mixed features. ${ }^{51}$

The expert consensus guidelines on depression with mixed features ${ }^{15}$ take a different approach and advise against the use of antidepressant monotherapy. Antidepressants are included in these guidelines only as potential third-line treatment options and only when used in conjunction with a mood stabilizer or atypical antipsychotic (eg, the combination of olanzapine and fluoxetine is a second-line option).

Thus, the selection of treatment for a patient with depression and mixed features revisits the debate 
disorders. ${ }^{53}$ A study by Frye et $\mathrm{al}^{50}$ included 176 adult patients with bipolar disorder who received 10 weeks of antidepressant treatment adjunctive to a mood stabilizer (possibly combined with an atypical antipsychotic) for a current depressive episode. The study showed that the co-occurrence of motor and verbal activation before patients received antidepressant treatment predicted emergent mania/hypomania $(P=.02)$. The potential for antidepressant treatment to destabilize patients with bipolar disorder was emphasized in a 2013 report by the International Society for Bipolar Disorders (ISBD).${ }^{54} \mathrm{Dr}$ McIntyre said that the ISBD convened a task force ${ }^{54}$ to review the use of antidepressants in bipolar disorders, and the task force concluded that, in many casesincluding cases in which the patient presents with mixed features-antidepressant treatment should be avoided because of insufficient evidence of benefit and the risk of mood destabilization. Dr McIntyre stated that some scenarios exist in which antidepressants can be used for individuals with bipolar disorder.

Evidence is lacking for antidepressant use in patients with MDD who exhibit mixed features. Prior to the introduction of the DSM-5 mixed features specifier, Koukopoulos and colleagues ${ }^{55}$ evaluated patients with depression and excitatory symptoms such as psychomotor agitation, talkativeness, racing thoughts, irritability, or mood lability. This study ${ }^{55}$ found that, for the majority of patients experiencing this type of mixed depression (56\%), the mixed symptoms emerged following pharmacologic treatment, primarily with antidepressants.

A more recent study by Sani et $\mathrm{al}^{56}$ used the same definition of mixed depression and came to a similar conclusion regarding antidepressants in this population. In about half of the patients in the study, mixed depression was associated with antidepressant use. In a different study, Patel and colleagues ${ }^{57}$ reviewed the electronic health records of over 21,000 adult patients receiving secondary mental health care for unipolar depression. The mean follow-up duration was 4.3 years. The investigators found a $10.9 \%$ rate of later diagnostic conversion to mania or a bipolar disorder. All antidepressants were associated with an increased incidence of mania in patients with unipolar depression, particularly selective serotonin reuptake inhibitors (95\% CI, 1.18-1.52) and venlafaxine (95\% CI, 1.07-1.70). ${ }^{57}$ Taken together, these studies provide evidence that antidepressants may be a dangerous treatment option for patients exhibiting or at risk for mixed features or conversion to bipolar disorder.

Among first-line treatment options, the Florida guideline states that mood stabilizers may be considered. ${ }^{51}$ The expert consensus guidelines, ${ }^{15}$ however, note that select atypical antipsychotics are the only drugs that have been specifically tested in depression with mixed features, and therefore these agents comprise stabilizers as second-line treatment. The evidence base for lithium as a treatment for depression with mixed features is limited, but it may help alleviate aggression or suicidality, and lamotrigine or divalproex may also be beneficial in mixed presentations. ${ }^{15}$

Atypical antipsychotics are among first-line treatment options provided by the Florida Medicaid guidelines ${ }^{51}$ and the only first-line treatment option recommended by the expert consensus guidelines. ${ }^{15}$ Some evidence is available supporting the efficacy of quetiapine, ${ }^{58}$ olanzapine, ${ }^{59}$ and asenapine ${ }^{60}$ in mood episodes with mixed features, but only ziprasidone ${ }^{61}$ and lurasidone ${ }^{62,63}$ have been assessed in patients with a diagnosis similar to that required by the DSM-5 for depression with mixed features.

Dr McIntyre described a study by Patkar and colleagues $^{61}$ that enrolled 73 patients who met DSM-IV criteria for a major depressive episode and who were also experiencing 2 or $3 D S M-I V$ manic criteria. Some patients had MDD, and some had bipolar II disorder. Participants were randomly assigned to receive either $40-160 \mathrm{mg} / \mathrm{d}$ of ziprasidone or placebo for 6 weeks. At the conclusion of the study, the active treatment group experienced a significantly greater improvement in depression scores as measured by the MontgomeryAsberg Depression Rating Scale $(P<.05)$, but more benefit was seen in bipolar II disorder than in MDD $(P<.05)$. No significant difference in mania rating scores was found for ziprasidone compared with the placebo group, which indicates that ziprasidone treatment did not lead to mood destabilization or switching in this sample. ${ }^{61}$

Dr McIntyre and his colleagues ${ }^{63}$ completed a post hoc analysis of a placebo-controlled trial investigating lurasidone for bipolar depression. The analysis found that among patients with mixed features, lurasidone was associated with significantly greater improvement in depression symptom ratings than was placebo $(P<.01)$. Rates of treatment-emergent mania or hypomania were similar (lurasidone, 2.2\%; placebo, 3.2\%). Similar results were obtained in a study by Suppes and colleagues, ${ }^{62}$ who conducted a placebo-controlled trial of lurasidone in 209 patients meeting DSM-IV-TR criteria for a major depressive episode with 2 or 3 manic symptoms. After 6 weeks of treatment, the lurasidone group had significantly greater improvement in both depression and manic symptoms and overall illness severity $(P<.001$ for all measures). ${ }^{62}$ The results of this study support lurasidone as a safe and effective treatment option for depression with mixed features.

According to the Florida Medicaid guidelines, ${ }^{51}$ all first-line treatments should be given a trial of at least 2 to 4 weeks. If the patient shows partial response, the treatment can be continued for an additional 4 weeks, or a level 2 treatment can be tried. If no response is evident at 2 to 4 weeks, a level 2 treatment should be tried. Level 2 
1 treatment, switching to monotherapy with a different second-generation antipsychotic or mood stabilizer, switching to an antidepressant monotherapy from the same or different class, or combining level 1 treatments. ${ }^{51}$ If the patient still fails to respond, third-level treatment options include electroconvulsive therapy, transcranial magnetic stimulation, alternative antidepressants such as tricyclics or monoamine oxidase inhibitors, or firstgeneration antipsychotics.

\section{Psychosocial Interventions}

Dr McIntyre pointed out that all patients with mood disorders should be considered for psychosocial treatment as an additional intervention. The Systematic Treatment Enhancement Program for Bipolar Disorder (STEP-BD) found that intensive psychosocial treatments such as cognitive-behavioral therapy, family-focused therapy, or interpersonal and social rhythm therapy as adjuncts to pharmacotherapy were helpful for enhancing stabilization from bipolar depression. ${ }^{64}$ Both guidelines ${ }^{15,51}$ include these interventions among treatment options. Furthermore, Dr McIntyre noted although lifestyle and behavioral modifications such as promoting healthy diet choices, good sleep hygiene, regular exercise, and smoking cessation have not been established as a means of improving mood symptoms in large, rigorous clinical trials, these interventions should nevertheless be discussed in patient visits due to their overall beneficial effects on health and quality of life. ${ }^{51}$

\section{Case Practice Question}

Discussion of the best response can be found at the end of the activity.

Case 3. Daniel is a 28-year-old man meeting criteria for a major depressive episode with mixed features. Which of the following treatments is not considered a first-line treatment option for Daniel according to the 2017 Florida Medicaid guidelines?

a. Conventional antidepressant

b. Lithium

c. Second-generation antipsychotic

d. Electroconvulsive therapy

\section{CONCLUSION}

To conclude, Dr McIntyre stated that mixed features are common in adults with MDD or bipolar disorder. Mixed features are associated with a more complex, severe, and chronic course as well as suboptimal treatment response. The available evidence is, in fact, insufficient to provide unequivocal recommendations. What evidence is available indicates that conventional antidepressants have been suboptimal in many cases, inviting the need for alternative treatment approaches. Emerging evidence suggests that some atypical antipsychotic agents may be effective in treating depressive episodes with mixed features. A descriptive literature hints at efficacy for conventional mood-stabilizing agents like lithium. Clinicians are encouraged to review the recent guidelines for additional support regarding sequencing treatments for adults with major depressive episodes and mixed features.

\section{Discussion of Case Practice Questions}

Case 1.

Preferred response: c. Sad mood

Because irritability, distractibility, and insomnia can be overlapping symptoms of a manic/hypomanic episode and a depressive episode, they are not on the list of symptoms needed for a mixed features specifier in a patient presenting with a manic or hypomanic episode. Sad mood is the only one of Juan's symptoms that is unique to a depressive episode and, therefore, may indicate the need for the mixed features specifier.

\section{Case 2.}

Preferred response: $d$. History of frequent suicidal thoughts

Anthony's history of suicidal ideation should raise suspicion for a potential bipolar diagnosis because suicidal thoughts and behaviors are more associated with mixed features and bipolar disorder than with MDD alone. Anthony should be evaluated regularly for hypomanic or manic symptoms.
Case 3.

Preferred response: $d$. Electroconvulsive therapy

The Florida Medicaid guidelines suggest antidepressants, lithium, or atypical antipsychotics as first-line treatment options in MDD with mixed features because all of these options have moodstabilizing properties. The guidelines do not introduce electroconvulsive therapy as a treatment option until a patient has failed at least 2 adequate trials of treatment with these mood-stabilizing agents.
Published online: September 26, 2017.

Disclosure of off-label usage: The authors have determined that, to the best of their knowledge, no drugs are approved by the US Food and Drug Administration for the treatment of a major depressive episode with mixed features.

\section{REFERENCES}

1. Marneros A, Goodwin FK. Bipolar disorders beyond major depression and euphoric mania. In: Marneros A, Goodwin FK, eds. Bipolar Disorders: Mixed States, Rapid Cycling, and Atypical Forms. New York, NY: Cambridge University Press; 2005:1-44.

2. Perugi G, Quaranta G, Dell'Osso L. The significance of mixed states in depression and mania. Curr Psychiatry Rep. 2014;16(10):486.

3. Ghaemi SN. Bipolar spectrum: a review of the concept and a vision for the future. Psychiatry Investig. 2013;10(3):218-224.

4. American Psychiatric Association. Diagnostic and Statistical Manual for Mental Disorders. Third Edition. Washington, DC: American Psychiatric Association; 1980.

5. American Psychiatric Association. Diagnostic and Statistical Manual for Mental Disorders. Fourth Edition, Text Revision. Washington, DC: American Psychiatric Association; 2000.

6. Malhi GS, Bargh DM, Cashman E, et al. The clinical management of bipolar disorder complexity using a stratified model. Bipolar Disord. 2012;14(suppl 2):66-89.

7. Machado-Vieira R, Luckenbaugh DA, Ballard ED, et al. Increased activity or energy as a primary criterion for the diagnosis of bipolar mania in DSM-5: findings from the STEP-BD study. Am J Psychiatry. 2017;174(1):70-76.

8. American Psychiatric Association. Diagnostic and Statistical Manual for Mental Disorders. Fifth Edition. Washington, DC: American Psychiatric 
9. Hu J, Mansur R, McIntyre RS. Mixed specifier for bipolar mania and depression: highlights of DSM-5 changes and implications for diagnosis and treatment in primary care. Prim Care Companion CNS Disord. 2014;16(2):doi:10.4088/PCC.13r01599.

10. Bauer MS, Simon GE, Ludman E, et al. "Bipolarity" in bipolar disorder: distribution of manic and depressive symptoms in a treated population. Br J Psychiatry. 2005;187(1):87-88.

11. Benazzi F. Is overactivity the core feature of hypomania in bipolar II disorder? Psychopathology. 2007;40(1):54-60.

12. Perugi G, Angst J, Azorin J-M, et al. Mixed features in patients with a major depressive episode: the BRIDGE-II-MIX study. J Clin Psychiatry. 2015;76(3):e351-e358.

13. Mclntyre RS, Soczynska JK, Cha DS, et al. The prevalence and illness characteristics of DSM-5-defined "mixed feature specifier" in adults with major depressive disorder and bipolar disorder: results from the International Mood Disorders Collaborative Project. J Affect Disord. 2015;172:259-264.

14. Smith DJ, Forty L, Russell E, et al. Sub-threshold manic symptoms in recurrent major depressive disorder are a marker for poor outcome. Acta Psychiatr Scand. 2009;119(4):325-329.

15. Stahl SM, Morrissette DA, Faedda G, et al. Guidelines for the recognition and management of mixed depression. CNS Spectr. 2017;22(2):203-219.

16. Targum SD, Suppes T, Pendergrass JC, et al. Major depressive disorder with subthreshold hypomania (mixed features): clinical characteristics of patients entered in a multiregional, placebo-controlled study. Prog Neuropsychopharmacol Biol Psychiatry. 2016;68:9-14.

17. Rush AJ, Trivedi MH, Ibrahim HM, et al. The 16-Item Quick Inventory of Depressive Symptomatology (QIDS), clinician rating (QIDS-C), and selfreport (QIDS-SR): a psychometric evaluation in patients with chronic major depression. Biol Psychiatry. 2003;54(5):573-583.

18. Zimmerman M, Chelminski I, McGlinchey JB, et al. A clinically useful depression outcome scale. Compr Psychiatry. 2008;49(2):131-140.

19. Zimmerman $M$, Chelminski I, Young D, et al. A clinically useful self-report measure of the DSM-5 mixed features specifier of major depressive disorder. J Affect Disord. 2014;168:357-362.

20. Berk M, Malhi GS, Cahill C, et al. The Bipolar Depression Rating Scale (BDRS): its development, validation and utility. Bipolar Disord. 2007;9(6):571-579.

21. Hirschfeld RMA. The Mood Disorder Questionnaire: a simple, patientrated screening instrument for bipolar disorder. Prim Care Companion J Clin Psychiatry. 2002;4(1):9-11.

22. Hirschfeld RM, Williams JB, Spitzer RL, et al. Development and validation of a screening instrument for bipolar spectrum disorder: the Mood Disorder Questionnaire. Am J Psychiatry. 2000;157(11):1873-1875.

23. Wang HR, Woo YS, Ahn HS, et al. The validity of the Mood Disorder Questionnaire for screening bipolar disorder: a meta-analysis. Depress Anxiety. 2015;32(7):527-538.

24. Nassir Ghaemi S, Miller CJ, Berv DA, et al. Sensitivity and specificity of a new bipolar spectrum diagnostic scale. J Affect Disord. 2005;84(2-3):273-277.

25. APA Work Group on Psychiatric Evaluation. In: Silverman JJ, Fochtmann LJ, Vergare MJ, et al, eds. The American Psychiatric Association Practice Guidelines for the Psychiatric Evaluation of Adults. 3rd ed. Arlington, VA American Psychiatric Association; 2016.

26. Vieta $\mathrm{E}$, Valentí M. Mixed states in DSM-5: implications for clinical care, education, and research. J Affect Disord. 2013;148(1):28-36.

27. Manning JS, Haykal RF, Connor PD, et al. On the nature of depressive and anxious states in a family practice setting: the high prevalence of bipolar II and related disorders in a cohort followed longitudinally. Compr Psychiatry. 1997;38(2):102-108.

28. Freedman $\mathrm{R}$, Lewis DA, Michels $\mathrm{R}$, et al. The initial field trials of DSM-5: new blooms and old thorns. Am J Psychiatry. 2013;170(1):1-5.

29. Regier DA, Narrow WE, Clarke DE, et al. DSM-5 field trials in the United States and Canada, part II: test-retest reliability of selected categorical diagnoses. Am J Psychiatry. 2013;170(1):59-70.

30. Hirschfeld RMA, Vornik LA, Lewis L. Perceptions and impact of bipolar disorder: how far have we really come? results of the National Depressive and Manic-Depressive Association 2000 Survey of individuals with bipolar disorder. J Clin Psychiatry. 2003;64(2):161-174.

31. Ratheesh A, Davey C, Hetrick S, et al. A systematic review and metaanalysis of prospective transition from major depression to bipolar disorder. Acta Psychiatr Scand. 2017;135(4):273-284.

32. Okasha T, Fikry M, Kowailed A, et al. Screening for bipolar disorder among patients undergoing a major depressive episode: report from the BRIDGE study in Egypt. J Affect Disord. 2013;147(1-3):217-224.

33. Judd LL, Akiskal HS, Schettler PJ, et al. The long-term natural history of the weekly symptomatic status of bipolar I disorder. Arch Gen Psychiatry.
34. Judd LL, Akiskal HS, Schettler PJ, et al. A prospective investigation of the natural history of the long-term weekly symptomatic status of bipolar II disorder. Arch Gen Psychiatry. 2003;60(3):261-269.

35. Gilman SE, Dupuy JM, Perlis RH. Risks for the transition from major depressive disorder to bipolar disorder in the National Epidemiologic Survey on Alcohol and Related Conditions. J Clin Psychiatry. 2012;73(6):829-836.

36. Bader CD, Dunner DL. Bipolar disorder not otherwise specified in relation to the bipolar spectrum. Bipolar Disord. 2007;9(8):860-867.

37. Robillard R, Naismith SL, Rogers NL, et al. Delayed sleep phase in young people with unipolar or bipolar affective disorders. J Affect Disord. 2013;145(2):260-263.

38. Oedegaard KJ, Syrstad VEG, Morken G, et al. A study of age at onset and affective temperaments in a Norwegian sample of patients with mood disorders. J Affect Disord. 2009;118(1-3):229-233.

39. Kawa I, Carter JD, Joyce PR, et al. Gender differences in bipolar disorder: age of onset, course, comorbidity, and symptom presentation. Bipolar Disord. 2005;7(2):119-125.

40. Sato T, Bottlender R, Sievers M, et al. Distinct seasonality of depressive episodes differentiates unipolar depressive patients with and without depressive mixed states. J Affect Disord. 2006;90(1):1-5.

41. Lee H-J, Son G-H, Geum D. Circadian rhythm hypotheses of mixed features, antidepressant treatment resistance, and manic switching in bipolar disorder. Psychiatry Investig. 2013;10(3):225-232.

42. Cassano GB, Rucci P, Frank E, et al. The mood spectrum in unipolar and bipolar disorder: arguments for a unitary approach. Am J Psychiatry. 2004;161(7):1264-1269.

43. Goldberg JF, Harrow M, Whiteside JE. Risk for bipolar illness in patients initially hospitalized for unipolar depression. Am J Psychiatry. 2001;158(8):1265-1270.

44. Tohen M, Khalsa H-MK, Salvatore P, et al. Two-year outcomes in firstepisode psychotic depression the McLean-Harvard First-Episode Project. J Affect Disord. 2012;136(1-2):1-8.

45. Mclntyre RS, Ng-Mak D, Chuang C-C, et al. Major depressive disorder with subthreshold hypomanic (mixed) features: a real-world assessment of treatment patterns and economic burden. J Affect Disord. 2017;210:332-337.

46. Akiskal HS, Benazzi F. Psychopathologic correlates of suicidal ideation in major depressive outpatients: is it all due to unrecognized (bipolar) depressive mixed states? Psychopathology. 2005;38(5):273-280.

47. Zimmermann P, Brückl T, Nocon A, et al. Heterogeneity of DSM-IV major depressive disorder as a consequence of subthreshold bipolarity. Arch Gen Psychiatry. 2009;66(12):1341.

48. Baldessarini RJ, Faedda GL, Offidani E, et al. Antidepressant-associated mood-switching and transition from unipolar major depression to bipolar disorder: a review. J Affect Disord. 2013;148(1):129-135.

49. Li C-T, Bai Y-M, Huang Y-L, et al. Association between antidepressant resistance in unipolar depression and subsequent bipolar disorder: cohort study. Br J Psychiatry. 2012;200(1):45-51.

50. Frye MA, Helleman G, McElroy SL, et al. Correlates of treatmentemergent mania associated with antidepressant treatment in bipolar depression. Am J Psychiatry. 2009;166(2):164-172.

51. Mclntyre RS, Suppes T, Tandon R, et al. Florida best practice psychotherapeutic medication guidelines for adults with major depressive disorder. J Clin Psychiatry. 2017;78(6):703-713.

52. Florida Medicaid Drug Therapy Management Program for Behavioral Health. 2015 Florida Best Practice Psychotherapeutic Medication Guidelines for Adults. University of South Florida. Sponsored by the Florida Agency for Health Care Administration. December 2015.

53. Vieta E. Antidepressants in bipolar I disorder: never as monotherapy. Am J Psychiatry. 2014;171(10):1023-1026.

54. Pacchiarotti I, Bond DJ, Baldessarini RJ, et al. The International Society for Bipolar Disorders (ISBD) task force report on antidepressant use in bipolar disorders. Am J Psychiatry. 2013;170(11):1249-1262.

55. Koukopoulos A, Sani G, Koukopoulos AE, et al. Melancholia agitata and mixed depression. Acta Psychiatr Scand suppl. 2007;115(433):50-57.

56. Sani G, Napoletano F, Vöhringer PA, et al. Mixed depression: clinical features and predictors of its onset associated with antidepressant use. Psychother Psychosom. 2014;83(4):213-221.

57. Patel R, Reiss $P$, Shetty $H$, et al. Do antidepressants increase the risk of mania and bipolar disorder in people with depression? a retrospective electronic case register cohort study. BMJ Open. 2015;5(12):e008341.

58. Suppes T, Ketter TA, Gwizdowski IS, et al. First controlled treatment trial of bipolar II hypomania with mixed symptoms: quetiapine versus placebo. J Affect Disord. 2013;150(1):37-43.

59. Tohen M, Kanba S, McIntyre RS, et al. Efficacy of olanzapine monotherapy in the treatment of bipolar depression with mixed 
60. Berk M, Tiller JWG, Zhao J, et al. Effects of asenapine in bipolar I patients meeting proxy criteria for moderate-to-severe mixed major depressive episodes: a post hoc analysis. J Clin Psychiatry. 2015;76(6):728-734.

61. Patkar A, Gilmer W, Pae C, et al. A 6 week randomized double-blind placebo-controlled trial of ziprasidone for the acute depressive mixed state. PLoS One. 2012;7(4):e34757.

62. Suppes T, Silva R, Cucchiaro J, et al. Lurasidone for the treatment of major depressive disorder with mixed features: a randomized, doubleblind, placebo-controlled study. Am J Psychiatry. 2016;173(4):400-407.

63. McIntyre RS, Cucchiaro J, Pikalov A, et al. Lurasidone in the treatment of bipolar depression with mixed (subsyndromal hypomanic) features: post hoc analysis of a randomized placebo-controlled trial. $J$ Clin Psychiatry. 2015;76(4):398-405.

64. Bowden $\mathrm{CL}$, Perlis $\mathrm{RH}$, Thase $\mathrm{ME}$, et al. Aims and results of the NIMH Systematic Treatment Enhancement Program for Bipolar Disorder (STEP-BD). CNS Neurosci Ther. 2012;18(3):243-249.

\section{POSTTEST}

To obtain credit, go to PSYCHIATRIST.COM (Keyword: October)

to take this Posttest and complete the Evaluation.

1. Ms A is a 22-year-old college senior who presents with depression, anxiety, and episodic bouts of severe insomnia. She meets the DSM-5 criteria for a major depressive episode. Among the following symptoms that are red flags suggesting that Ashley might meet criteria for the mixed features specifier, all are DSM-5 criteria except:

a. Racing thoughts/flight of ideas

b. Increase in energy

c. Distractibility

d. Decreased need for sleep

2. Mrs $\mathrm{J}$ is a 47-year-old woman presenting with depressed mood, loss of interest, hypersomnolence, excessive eating, fatigue, and difficulties with concentration lasting 5 weeks. Additionally, she reports racing thoughts, irritability, and occasional periods when she requires very little sleep lasting 2 to 3 days. Her speech is pressured. Which statement about her mood state is false?

a. Her depression with mixed features can be a part of major depressive disorder (MDD) or bipolar II disorder

b. Depressive episodes with mixed features are associated with a later onset of disease

c. Presence of a depressive episode with mixed features may predict a transition from MDD to bipolar disorder

d. Her presentation does not exclude a possible diagnosis of bipolar I disorder

3. You have diagnosed Ms $\mathrm{N}$ with MDD and mixed features. To provide first-line psychopharmacologic treatment according to both the Florida Medicare guidelines and recent expert consensus guidelines, which of the following therapeutic choices would be best for Ms N?
a. Benzodiazepine
b. Psychostimulant
c. Select second-generation antipsychotic
d. Antidepressant 